\title{
DEFECT AND DOPANT DEPTH PROFILES IN BORON-IMPLANTED SILICON STUDIED WITH CHANNELING AND NUCLEAR REACTION ANALYSIS
}

\author{
M. VOS, D.O. BOERMA and P.J.M. SMULDERS \\ Laboratorium voor Algemene Natuurkunde and Materials Science Centre, Rijksuniversiteit Groningen, Groningen, The Netherlands
}

\author{
S. OOSTERHOFF \\ Department of Electrical Engineering, Twente University of Technology, Enschede, The Netherlands
}

Received 21 May 1986

\begin{abstract}
Single crystals of silicon were implanted at RT with $1 \mathrm{MeV}$ boron ions to a dose of $1 \times 10^{15}$ ions $/ \mathrm{cm}^{2}$. The depth profile of the boron was measured using the $2060-\mathrm{keV}$ resonance of the ${ }^{11} \mathrm{~B}(\alpha, \mathrm{n}){ }^{14} \mathrm{~N}$ nuclear reaction. The distribution of the lattice disorder as a function of depth was determined from channeling of $\mathrm{MeV} \alpha$-particles. This was done in the as-implanted case and after furnace annealing at temperatures up to $1000^{\circ} \mathrm{C}$. A short description of the applied techniques is presented. The crystal disorder was found to almost vanish during annealing at $600^{\circ} \mathrm{C}$ and to reappear at higher annealing temperatures at a depth coinciding with the projected range of the boron ions. Both the boron and the disorder depth profiles are broadened after annealing at $1000^{\circ} \mathrm{C}$. The results agree with recent findings on defect annealing in silicon.
\end{abstract}

\section{Introduction}

During the last few years much experimental work on $\mathrm{MeV}$ implantations in semiconductors has been reported [1,2]. High-energy implantation offer the possibility to make deep junctions or buried layers. A drawback of the implantation technique is the introduction of lattice disorder. A well defined anneal treatment is necessary in order to reduce the disorder and to retain the implantation profile [3]. A detailed understanding of the annealing process and accurate data on the depth profiles after implantation and annealing are indispensible for the fabrication process.

Measurement of the depth profile of the implanted dopant with Rutherford backscattering (RBS) or (resonant) nuclear reaction analysis (NRA) yields absolute scales for the depth and the concentration and may serve as a calibration for other methods, provided the stopping powers of the particles used are well known. The measurement of the depth distribution of lattice disorder with RBS combined with channeling is very direct and is an important addition to the existing methods. In the case of deep inplants it is possible to distinguish between disorder which is related to the implanted dopant and disorder in impurity-free parts of the crystal.

In the present study the boron depth profile of ${ }^{11} \mathrm{~B}$-implanted monocrystalline silicon wafers is measured using the ${ }^{11} \mathrm{~B}(\alpha, \mathrm{n}){ }^{14} \mathrm{~N}$ nuclear reaction. To our knowledge it is the first time that this resonant reaction is used for depth profiling. For this reason, the experimental technique will be described in some detail in section 2.2 .

With the same samples the depth profile of lattice disorder was determined from channeling of $\mathrm{MeV} \alpha-$ particles. The method has been described in the literature [4]. Therefore, only a short resumé of the method will be given in section 2.3 . The measurements were performed on the as-implanted samples and after furnace annealing at $600,750,900$ and $1000^{\circ} \mathrm{C}$. The results, presented in section 3 , are compared with existing data and theories in section 4 .

\section{Experimental techniques}

\subsection{Sample preparation}

Single crystals of p-type silicon with a $\langle 100\rangle$ orientation were implanted at RT with $1 \mathrm{MeV} \mathrm{B}^{2+}$ ions to a dose of $1 \times 10^{15}$ ions $/ \mathrm{cm}^{2}$. This was done using the 500 kV HVEE implanter of the Twente University of Technology. The implantation direction was tilted $7^{\circ}$ from $\langle 100\rangle$ and rotated $27^{\circ}$ from a (100) plane to suppress channeling to a minimum. The beam heating during implantation was estimated to be a few degrees only, due to the low beam current of $-50 \mathrm{nA} / \mathrm{cm}^{2}$. The annealings were done in a dry $\mathrm{N}_{2}$ atmosphere. In the case of $900^{\circ} \mathrm{C}$ annealing it was checked that there was no difference between a vacuum-annealed sample and a 
sample annealed in a nitrogen atmosphere. After annealing at $1000^{\circ} \mathrm{C}$ an oxide layer of about $10 \mathrm{~nm}$ was observed with RBS. The layer was removed in a dilute HF solution before the depth profiling of the boron and of the damage. The profilings were carried out using $\mathrm{He}^{+}$ions from the Groningen 6 MV Van de Graaff accelerator.

\subsection{Depth profiling of boron with the ${ }^{11} B(\alpha, n){ }^{14} \mathrm{~N}$ reac- tion}

The reaction ${ }^{11} \mathrm{~B}(\alpha, \mathrm{n}){ }^{14} \mathrm{~N}$ shows some marked resonances in the energy region $E_{\alpha}=1.5-3 \mathrm{MeV}$ [5]. The strong resonance at $E_{\alpha}=2.06 \mathrm{MeV}$ looks promising for depth profiling as there are no neighbouring resonances within a few hundreds of $\mathrm{keV}$. The $Q$-value of the reaction is $0.1574 \mathrm{MeV}$, resulting in a neutron energy of $2.07 \mathrm{MeV}$ at $0^{\circ}$ for the $E_{\alpha}=2.06 \mathrm{MeV}$ resonance. Possible disturbing reactions are the ${ }^{13} \mathrm{C}(\alpha, n){ }^{16} \mathrm{O}$ and ${ }^{17,18} \mathrm{O}(\alpha, \mathrm{n}){ }^{20,21} \mathrm{Ne}$ reactions which also have positive $Q$ values and are known to have comparable neutron yields [6]. The ${ }^{17} \mathrm{O}$ and ${ }^{18} \mathrm{O}$ reactions would only be a problem if the bulk material contained a large fraction of oxygen. When care is taken to avoid carbon buildup, the ${ }^{13} \mathrm{C}(\alpha, n){ }^{16} \mathrm{O}$ background may also be reduced to a negligible level. Under these conditions there is no need for a high resolution neutron spectrometer and an organic-scintillator detector may be used. The scintillation response of such detectors shows a rapidly decaying component, and a slow component, with different ratios for gammas and neutrons. This enables the use of pulse shape discrimination between neutrons and gammas.

The targets were mounted in a special vacuum chamber pumped to less than $10^{-7}$ Torr. No carbon buildup was observed with this system, even after several hours of $\mathrm{He}^{+}$bombardment, neither in the observed yield, nor by visual inspection of the samples. A diaphragm with an aperture of $5 \mathrm{~mm}$ was situated $5 \mathrm{~cm}$ in front of the target. Care was taken that this diaphragm received less than a few percent of the beam current. The next but last diaphragm, with a smaller diameter, was situated 2 $m$ in front of the target chamber and well shielded by lead and boron-paraffin. The detector, placed at $0^{\circ}$ about $3 \mathrm{~cm}$ from the target, consisted of a $100 \mathrm{ml}$ cell of NE213 scintillation liquid mounted on a RCA8575 photomultiplier. The anode pulses were fed to a Canberra 2160 pulse shape discriminator [7] and also to an Ortec 463 constant fraction discriminator. The time difference between the output pulses of these two circuits was digitalized, as well as an energy-proportional signal taken from the 9 th dynode of the photomultiplier. The degree of discimination between neutrons and gammas was very satisfactory as illustrated in fig. 1 , showing a two-parameter time-energy spectrum obtained during an actual depth profiling measurement. For the yield

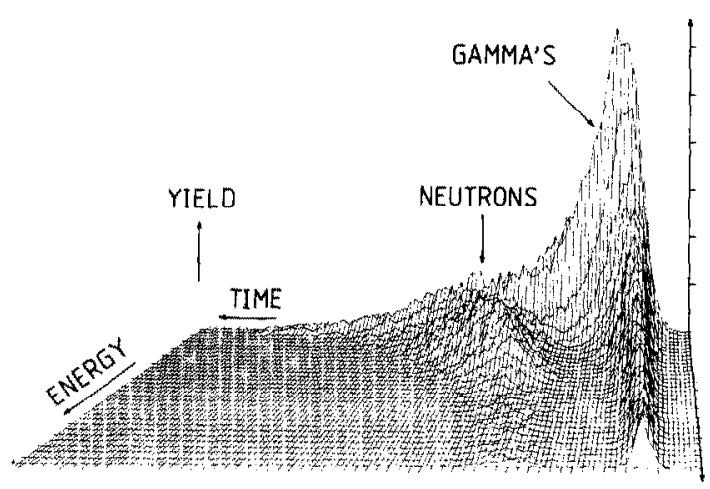

Fig. 1. Spectrum of pulses from the neutron detector, sorted as function of the two parameters, pulse height (= energy) and pulse shape $(=$ time). This spectrum was obtained during a yield curve measurement of a boron-implanted silicon sample.

curves to be discussed events were counted of which the energy and time signals each fell within a window set on the respective pulse height distribution.

Fig. 2 shows the neutron yield obtained by bombarding a $13 \mu \mathrm{g} / \mathrm{cm}^{2}$ B target evaporated onto a $\mathrm{Cu}$ backing. The vertical scale represents the number of counts per $50 \mu \mathrm{C}$. integrated beam current within a neutron-energy window optimal for the $2060 \mathrm{keV}$ resonance. This means that for higher bombarding energies the high energy part of the neutron spectrum is cut off. The resonances at $E_{\alpha}=1510,1580,2060$ and $2610 \mathrm{keV}$ may be identified as known ${ }^{11} \mathrm{~B}(\alpha, n){ }^{14} \mathrm{~N}$ resonances $[5,8]$.

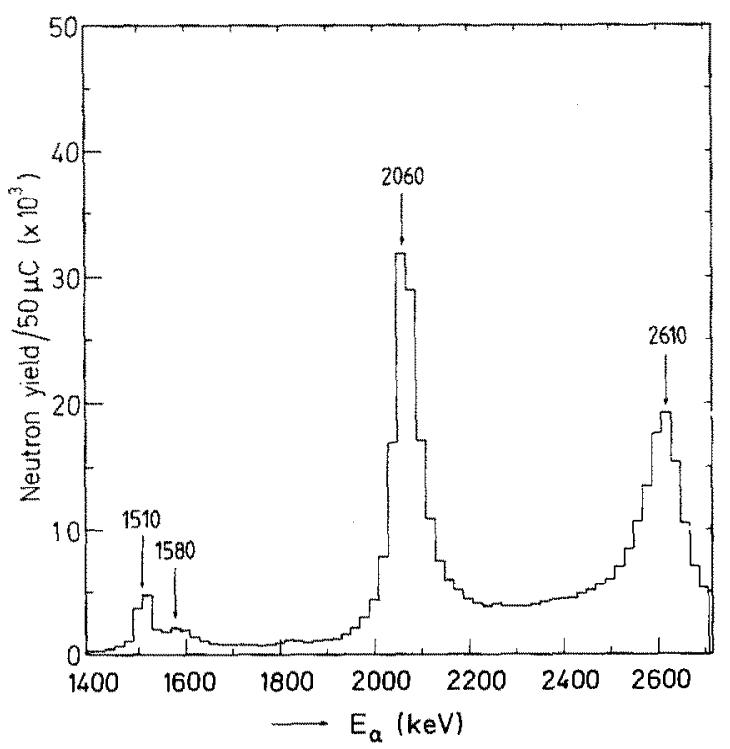

Fig. 2. Yield curve of neutrons from the reaction ${ }^{11} \mathrm{~B}(\alpha, \mathrm{n}){ }^{14} \mathrm{~N}$ in the energy region $E_{\alpha}=1400-2700 \mathrm{keV}$. Target: $13 \mu \mathrm{g} / \mathrm{cm}^{2}$, natural boron. 
The width of the $2060 \mathrm{keV}$ resonance, deduced from a more detailed measurement than shown in fig. 2, was found to be $\Gamma_{\mathrm{lab}}=45 \pm 3 \mathrm{keV}$, or $\Gamma_{\mathrm{cm}}=33 \pm 2 \mathrm{keV}$, in good agreement with Van der Zwan and Geiger [5]. This limits the depth resolution for profiling of $\mathrm{B}$ in $\mathrm{Si}$, where the stopping power of $2.1 \mathrm{MeV}$ alpha particles is $224 \mathrm{eV} / \mathrm{nm}$ [9], to a value of the order of $200 \mathrm{~nm}$. However, the range can be as large as $2 \mu \mathrm{m}$.

For the near-surface region the resonances at 2941 and $2980 \mathrm{keV}$ bombarding energy may be of interest. The yield in the region $E_{\alpha}=2900-3015 \mathrm{keV}$ is shown in fig. 3 measured under somewhat different conditions than fig. 2 with a target of only $5 \mu \mathrm{g} / \mathrm{cm}^{2}$, and $10 \mu \mathrm{C}$ beam dose per point. The width of the resonances was found to be $\Gamma_{\mathrm{lab}}=4.5 \pm 0.3 \mathrm{keV}\left(E_{\alpha}=2941 \mathrm{keV}\right)$ and $\Gamma_{\text {lab }}=6.5 \pm 0.3 \mathrm{keV}\left(E_{\alpha}=2980 \mathrm{keV}\right)$, somewhat lower than the literature values [5]. If these resonances were used the depth resolution would be mainly determined by the energy straggling. However, the high level of continuous background under the resonances and their close proximity would make the unambiguous determination of depth profiles deeper than about $200 \mathrm{~nm}$ very difficult. Therefore we concentrated on the $E_{\alpha}=$ $2060 \mathrm{keV}$ resonance.

For each sample the yield as a function of bombarding energy was measured. The actual depth profiles were extracted from these measurements using a deconvolution procedure recently described [10] in which the thin target yield (fig. 2 ) is used as response function and the straggling is taken into account.

\subsection{Depth profiling of damage}

For damage depth profiling several spectra of scattered particles (in this case $3 \mathrm{MeV} \alpha$-particles) are

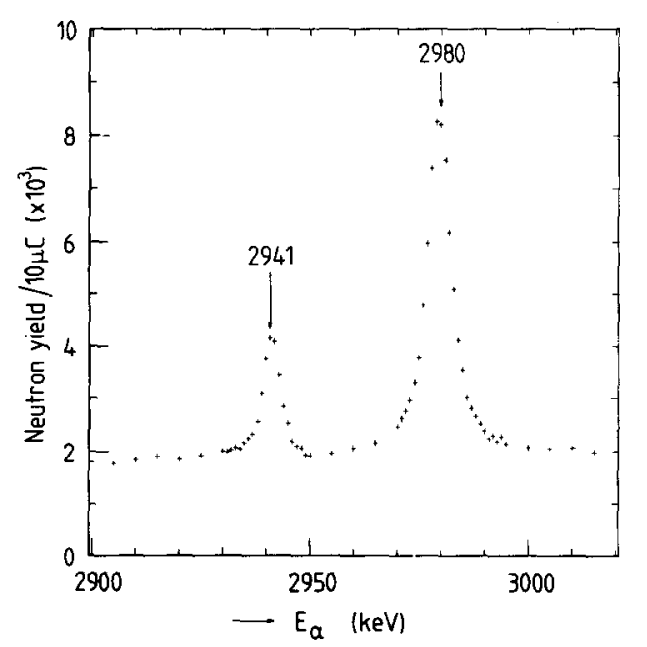

Fig. 3. Yield curve of neutrons from the reaction ${ }^{11} B(\alpha, n){ }^{14} N$ in the region $E_{\alpha}=2900-3015 \mathrm{keV}$. measured with the beam direction coinciding with an axial or planar (channeling) direction or with a "random" direction. From these data the normalized yields $\chi(z)$ are derived by dividing the channeled yield from depth $z$ by the random yield from the same depth. This is done for damaged (implanted) and undamaged (virgin) parts of the same crystal. Random stopping powers are used to obtain the depth scale for the yields, both for the incoming and outgoing trajectories of the scattered particles. This point will be discussed later. The normalized yield of a damaged crystal is given by [4]:

$\chi_{\mathrm{d}}(z)=\chi_{\mathrm{R}}(z)+\left(1-\chi_{\mathrm{R}}(z)\right) f n_{\mathrm{d}}(z)$,

Here $\chi_{R}(z)$ is the dechanneled (random) fraction at depth $z$. The second term describes the direct scattering of channeled particles by a number $n_{\mathrm{d}}$ of displaced atoms per unit volume with a scattering factor $f$.

The first term $\chi_{R}(z)$ describes the particles entering the detector after dechanneling: first scattering brings the particle form a channeling trajectory to a random trajectory; then the random particle may be scattered into the detector. The dechanneled fraction can be expressed as:

$$
\begin{aligned}
\chi_{\mathrm{R}}(z)= & \chi_{\mathrm{v}}(z)+\left[1-\chi_{\mathrm{v}}(z)\right] \\
& \times\left[1-\exp \left(-\int_{0}^{z} \sigma_{\mathrm{d}} n_{\mathrm{d}}\left(z^{\prime}\right) \mathrm{d} z^{\prime}\right)\right]
\end{aligned}
$$

with $\chi_{\mathrm{v}}(z)$ the normalized yield for a virgin part of the crystal and $\sigma_{d}$ the efficiency for dechanneling by a defect. If the direct scattering factor is zero, one obtaines the integrated dechanneling probability $I(z)$ :

$I(z)=\int_{0}^{z} \sigma_{d} n_{d}\left(z^{\prime}\right) \mathrm{d} z^{\prime}=-\log \frac{\left(1-\chi_{\mathrm{d}}(z)\right)}{\left(1-\chi_{\mathrm{V}}(z)\right)}$,

The damage profile $\sigma_{\mathrm{d}} n_{\mathrm{d}}$ is obtained by calculating $\mathrm{d} I(z) / \mathrm{d} z$. If both dechanneling and direct scattering are important eq. (1) is most easily solved for the axial case. Then $1-\chi(\mathrm{z}) \cong 1$ and $\int_{0}^{z} \sigma_{\mathrm{d}} n_{\mathrm{d}}\left(z^{\prime}\right) \mathrm{d} z^{\prime} \ll 1$. Eqs. (1) and (2) reduce to

$\chi_{\mathrm{d}}(z)-\chi_{\mathrm{v}}(z)=\int_{0}^{z} \sigma_{d} n_{d}\left(z^{\prime}\right) \mathrm{d} z^{\prime}+f n_{\mathrm{d}}(z)$

One can obtain the damage profile $\sigma_{\mathrm{d}} n_{\mathrm{d}}(z)$ by choosing $f$ in such a way that the damage level goes to zero for large depth.

For (small clusters of) interstitials the direct scattering contribution is important. For dislocation loops the direct scattering factor is zero and the dechanneling cross section $\sigma_{\mathrm{d}}$ is proportional to $\sqrt{\left(E / Z_{1}\right)}$ [11], with $E$ the energy, and $Z_{1}$ the atomic number of the incoming particles. Thus channeling provides information about the depth distribution and type of the defects but no detailed information on the size of defects, Burgers vector of dislocations, etc. 


\section{Results}

The boron concentration as a function of depth measured using the $2060 \mathrm{keV}$ resonance of the ${ }^{11} \mathrm{~B}(\alpha, n){ }^{14} \mathrm{~N}$ reaction is shown in fig. 4 . The profile was obtained as described in section 2.2. All conversions from energy to depth scale were made using the stopping power function and parameter values as given by Ziegler [9]. The result is a mean projected range $R_{\mathrm{p}}=$ $1640 \mathrm{~nm}$ and an upper limit of the width of the distribution of $200 \mathrm{~nm}$ fwhm.
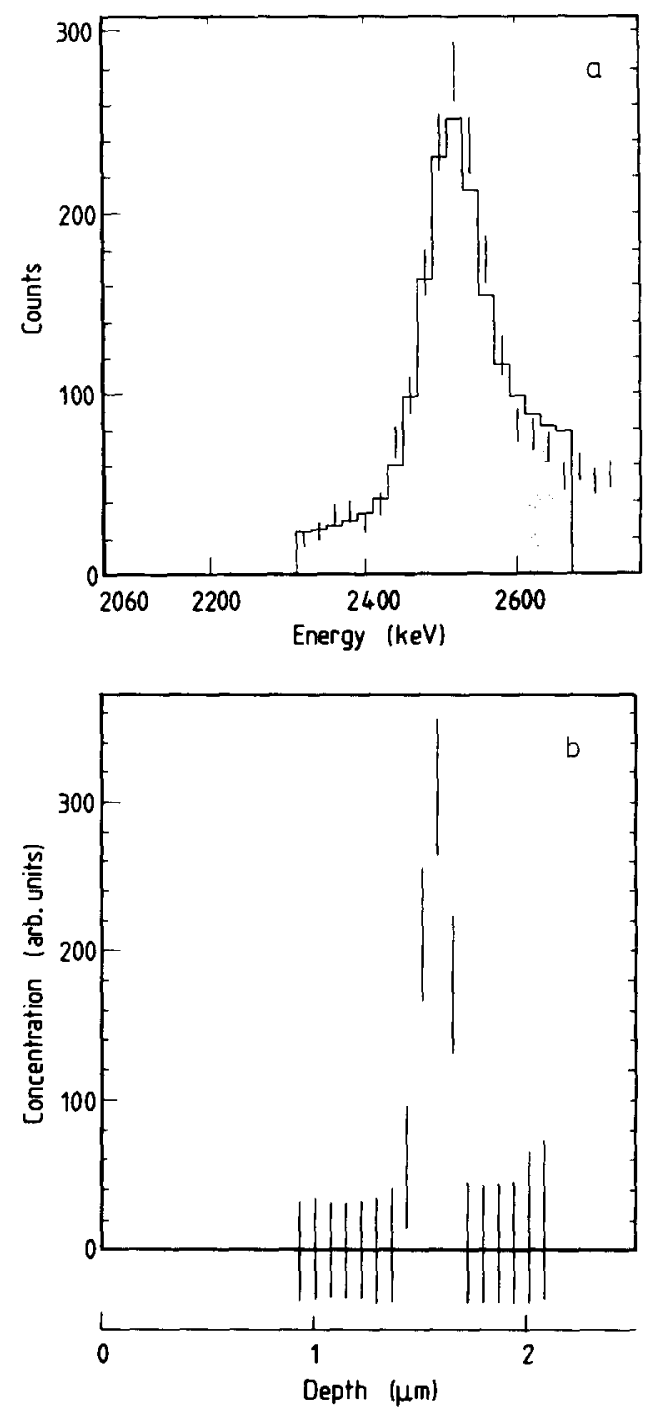

Fig. 4. (a) Neutron yield curve for a silicon crystal implanted with $1 \mathrm{MeV} \mathrm{B}^{2+}$ ions to a dose of $10^{15} \mathrm{at} . / \mathrm{cm}^{2}$. The solid line is the fitted curve for the depth profile shown in (b). (b) Depth distribution of boron atoms calculated by deconvolution of the yield curve of (a) (see text).
In fig. $5\langle 100\rangle$ axial and (110) planar channeling spectra measured. after implantation and subsequent annealing are shown together with the spectra taken at the unimplanted part. After implantation the spectra show a direct scattering peak, indicating that the detected damage consists of (small clusters of ) interstitials. The damage profile shown in fig. 6 was calculated from the axial spectra with eq. (4). A simple model would predict a damage level proportional to the energy deposited in nuclear stopping by the implanted boron ions. The depth distribution of this deposited energy has been calculated by Brice [12] and is also depicted in fig. 6. This distribution has been scaled such that the calculated projected range of $1534 \mathrm{~nm}$ coincides with the experimental value of $1640 \mathrm{~nm}$. In this way qualitative agreement is found between the measured profile and the calculation.

After annealing at $600^{\circ} \mathrm{C}$ the channeling spectra of the implanted and unimplanted part show no difference within the limits of error, indicating that the damage, visible with the applied method, has vanished.

As can be seen in fig. 5, disorder is observed again after annealing at $750^{\circ} \mathrm{C}$. The spectra show an enhanced dechanneling at a depth roughly coinciding with the projected range of the boron. After annealing at $900^{\circ} \mathrm{C}$ the dechanneling in the region is even stronger. Annealing at $1000^{\circ} \mathrm{C}$ causes a decrease of the dechanneling probability and a broadening of the disordered zone.

The spectra of the annealed sample show no evidence for direct scattering. Therefore the depth distributions of the disorder, the damage depth profiles, were calculated by differentiating a spline fit of the integrated dechanneling probability as given by eq. (3). The damage profiles obtained from axial and planar channeling spectra are compared in fig. 7. In the profile obtained from planar channeling spectra the disorder level returns to zero at a depth larger than $1800 \mathrm{~nm}$. However, the disorder seems to extend much deeper in the profile derived from axial channeling spectra. This is attributed to multiple scattering effects not contained in the simple theory used. For the planar case the theory used seems to work surprisingly well. Note that the maximum of the damage is found at the same depth for planar and axial channeling. In the following, only damage profiles obtained from planar channeling spectra are considered.

These damage depth profiles are shown in fig. 8 together with the depth distributions of the boron. The boron profile is clearly broadened after annealing at $1000^{\circ} \mathrm{C}$; no change with respect to the as-implanted case was observed after annealing at temperatures up to $900^{\circ} \mathrm{C}$. The boron depth profiles were found at a slightly larger depth than the damage depth profiles. The difference may be caused by the use of random stopping powers to obtain the depth scales from the channeled 

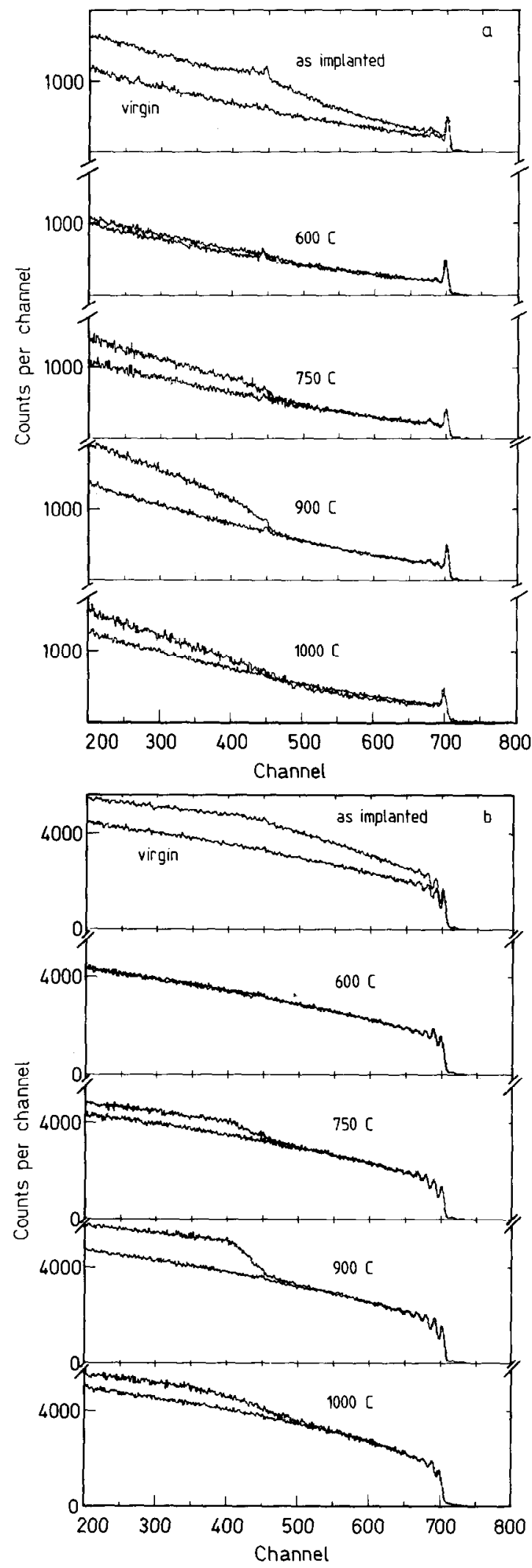

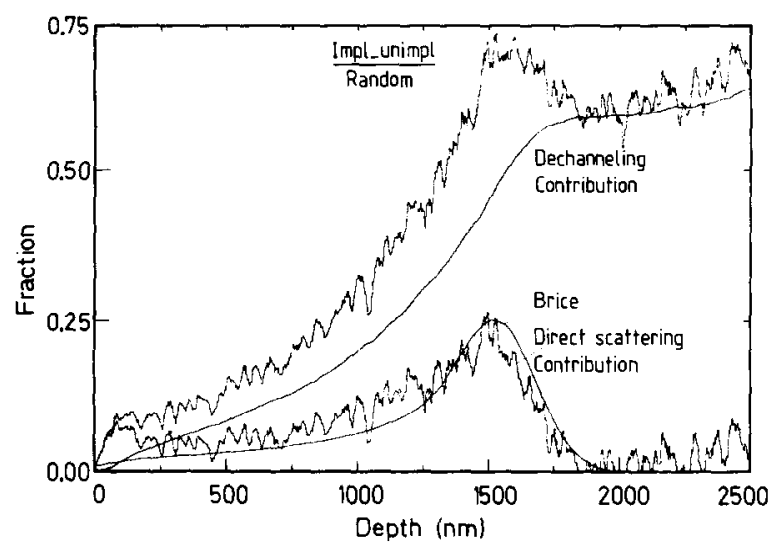

Fig. 6. Normalized difference between the scattering yield as a function of depth from an implanted and an unimplanted part of the Si crystal (top curve). The decomposition into a dechanneling and a direct scattering contribution is also indicated. The direct scattering contribution is compared with the shape of the energy deposition density as calculated by Brice [12].

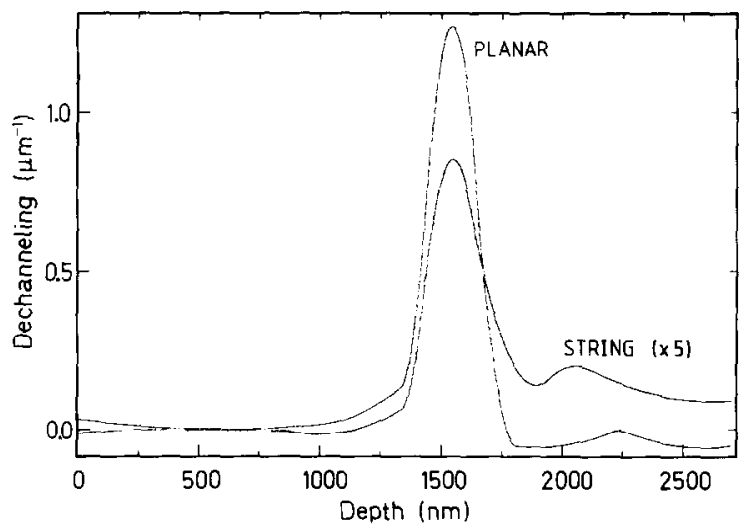

Fig. 7. Depth profiles of damage extracted from axial and planar channeling spectra for a sample annealed at $900^{\circ} \mathrm{C}$.

spectra. In reality the average stopping power of the $\alpha$-particles penetrating the crystal in a planar or axial channeling direction is reduced. For perfectly channeled particles in axial direction the reduction can amount to $50 \%$ [13], in planar direction the reduction is in the order of $10 \%$. In the axial case only a small fraction of the particles dechannels due to defects. Obviously these

Fig. 5. Energy spectra of $3 \mathrm{MeV} \alpha$-particles scattered from silicon implanted with $1 \mathrm{MeV}$ boron ions $\left(10^{15} / \mathrm{cm}^{2}\right)$. The spectra are taken after annealing at temperatures as indicated. Channeling conditions: (a) beam direction along the $\langle 100\rangle$ string. (b) beam direction in the (110) plane Each figure also shows the spectrum obtained from an unimplanted (virgin) part of the sample. 


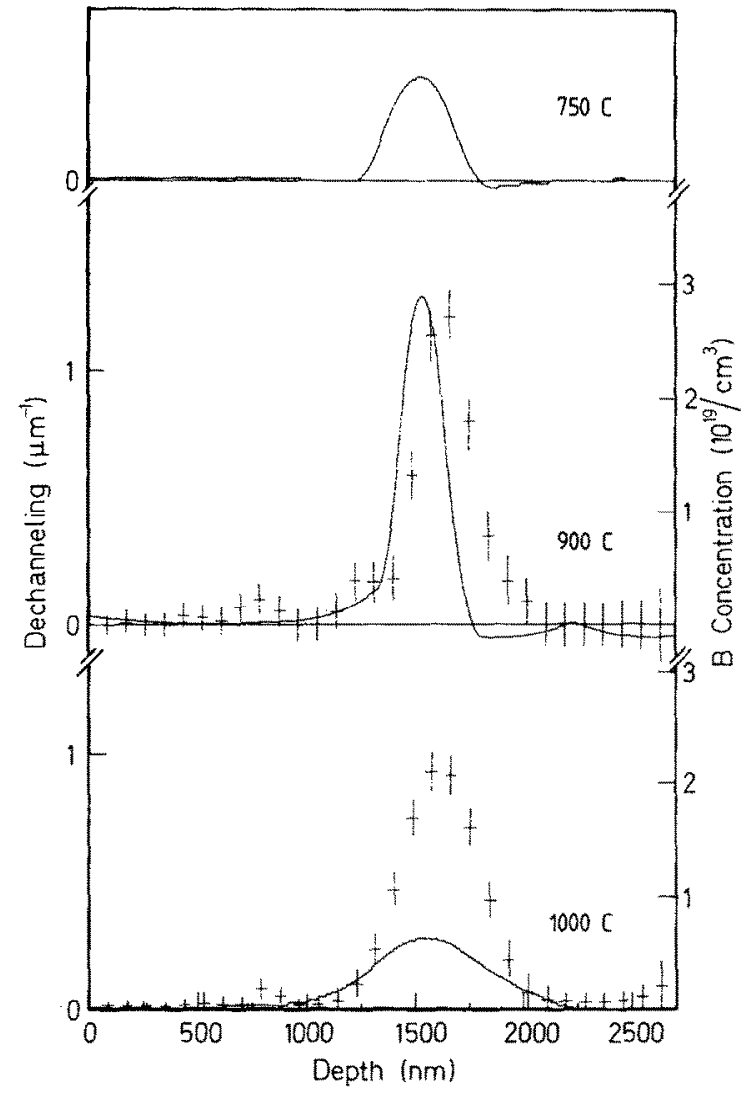

Fig. 8. Depth profiles of damage derived from planar channeling spectra taken after annealing at different temperatures (solid curves). Boron depth profiles (vertical bars) are given for comparison.

particles will be the poorly channeled ones which experience a stopping power close to the random value. This is the usual argument to justify the use of random stopping powers [14]. Because the damage is very deep in the present case, this reasoning may not be fully applicable. In the planar case a larger fraction of some $25 \%$ dechannels due to the damage. The stopping power for these particles may be closer to the average planar value. If the mismatch of the damage and boron depth profiles is indeed due to the stopping power effects described, it is a coincidence that the damage is observed at the same depth in the axial and planar cases (see fig. 7). A reduction by about $10 \%$ of the effective energy loss of both planar and axial channeled particles would solve the discrepancy between the boron concentration and damage profiles.

To get an impression of the influence of a modification of the stopping power on the projected range values, and on the discrepancy discussed, all depth profiles were recalculated with a depth scale derived

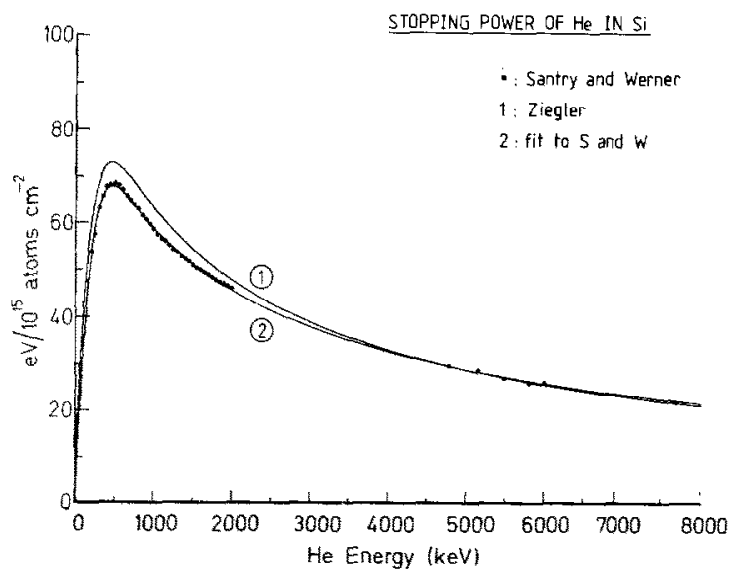

Fig. 9. Stopping power of $\mathrm{He}$ in Si. Data points from Santry and Werner $[15,16]$. The solid lines are: 1) the function used by Ziegler [9] to describe the stopping power, and 2) the same function with modified coefficients, $A=\{1.004,0.768$, $62.582,0.771,1.99\}$, providing a smooth interpolation through the data points.

from recent stopping power values for $\mathrm{He}$ in $\mathrm{Si}$, measured by Santry and Werner $[15,16]$. The data of these authors were fitted to a functional form as given by Ziegler [9], see fig. 9. Using this function the difference between the mean ranges of boron and of the damage reduces from $70 \mathrm{~nm}$ to $58 \mathrm{~nm}$, while the projected range of boron increases from $1640 \mathrm{~nm}$ to $1710 \mathrm{~nm}$. It should also be mentioned that different parts of the stopping power curves (fig. 9) are used to obtain the depth of the damage and of the boron. This may lead also to a discrepancy in the depths found. For the NRA measur-

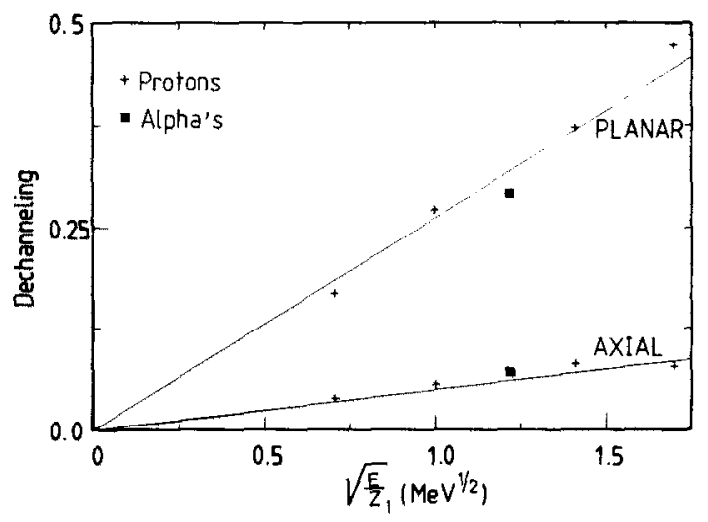

Fig. 10. The integrated dechanneling probability $I$ at a depth of $2000 \mathrm{~nm}$ as a function of $\left(E / Z_{1}\right)^{1 / 2}$. Shown are experimental data fitted with a straight line for planar channeling (upper curve) and axial channeling (lower curve). Data points obtained with $\alpha$-particles and protons as indicated. 
ments yielding the boron depths the stopping power between 2 and $2.5 \mathrm{MeV}$ is used. The depth scale for the damage is derived from channeling spectra using the stopping power from 2.7 to $3.0 \mathrm{MeV}$ (incoming trajectory) and from 1.0 to $1.6 \mathrm{MeV}$ (outgoing trajectory).

In a separate series of experiments the energy dependence of the integrated dechanneling probability was measured using protons as well as $\alpha$-particles for the sample annealed at $900^{\circ} \mathrm{C}$. The results obtained for the planar (110) as well as for the axial $\langle 100\rangle$ cases are shown in fig. 10. It was found that the dechanneling probability is proportional to $\left(E / Z_{1}\right)^{1 / 2}$ for values of $E / Z_{1}$ at least up to $3 \mathrm{MeV} / Z_{1}$. From this it is concluded that the dechanneling is caused by dislocations or dislocation loops with a diameter $>50 \mathrm{~nm}$ as estimated using the calculations of Kudo [11].

\section{Discussion}

The value found for the average projected range of 1 $\mathrm{MeV}$ boron in Si is $1640 \mathrm{~nm}$ or $1710 \mathrm{~nm}$, depending on the set of stopping parameters used. The estimated statistical error is $20 \mathrm{~nm}$, which means that the total error is detemined mainly by the uncertainty in the stopping power. The projected range values are close to the value of $1670 \mathrm{~nm}$ determined with the $\mathrm{CV}$ method as well as with SIMS [17]. The broadening of the boron depth profile at $1000^{\circ} \mathrm{C}$ is in agreement with the findings of others, see ref. [18], for instance.

The development of the damage profile as a function of annealing temperature can be understood if a differentiation is made between the intrinsic damage in the silicon caused by the energy deposited in nuclear collisions and damage which is associated with the boron impurities. As has been pointed out by Narayan and Fletcher [19], the intrinsic damage resembles the damage caused by fast neutron irradiation. During neutron irradiations the recoiling silicon atoms give rise to nonamorphizing damage cascades as in the case of boron implantation. After neutron irradiation interstitial clusters with 6-18 atoms and smaller vacancy clusters containing some 3 vacancies were observed with $\mathrm{X}$-ray diffuse scattering [20]. In addition, small interstitial loops with an average size of $28 \AA$ and even smaller vacancy loops with an average size of the visible loops of $7 \AA$ were observed with TEM and X-ray diffuse scattering $[19,21]$. The dechanneling by the vacancy type of defects probably is very small. The small interstitial type of defects contain silicon atoms in interstitial positions. This would explain the large direct scattering component after the boron implantation observed in this work as well as in other studies [22]. From references $[19,20]$ it is known that the intrinsic damage anneals out in a number of anneal steps and completely vanishes at anneal temperatures between $600^{\circ} \mathrm{C}$ and $700^{\circ} \mathrm{C}$, depending on the density of the damage. This is in agreement with the fact that we see almost no damage after annealing at $600^{\circ} \mathrm{C}$.

From channeling studies [23] it is known that after implantation with $10^{15}$ ions $/ \mathrm{cm}^{2}$ and annealing at $600^{\circ} \mathrm{C}$ the boron atoms occupy mainly random lattice sites. After annealing at temperatures between $600^{\circ} \mathrm{C}$ and $1000^{\circ} \mathrm{C}$ the substitutional fraction increases gradually to $90 \%$. The dissolution of the boron causes a relatively large lattice dilatation of $(1 / C)(\Delta a / a)=0.26$ [24] with a fractional concentration $C$ and lattice constant $a$. The strain caused by the dilatation can induce dislocation loops. It has also been suggested [19] that interstitial atoms, created by a kick-out mechanism when the boron dissolves, cluster to create interstitial type of dislocation loops. One or both of the mechanisms may cause the reappearance of damage observed approximately at the depth of the implanted boron. In TEM work $[19,25,26]$ this damage in regions of high boron concentration has been seen in the form of characteristic rod-shaped defects and (interstitial type of) dislocation loops. The rod-shaped defects, which may contain boron atoms, anneal out at $750^{\circ} \mathrm{C}$. The dislocations coarsen and only anneal out above $900-1000^{\circ} \mathrm{C}$. This picture is in agreement with the present findings. It explains the fact that the dechanneling after annealing at $900^{\circ} \mathrm{C}$ is mainly caused by dislocations or dislocation loops.

The broadening of the damage profile together with the boron profile after $1000^{\circ} \mathrm{C}$ annealing has not been reported before. It may be caused by the creation of new damage by one of the mechanisms mentioned above or it may be due to the pinning of moving dislocations by boron atoms.

This work is part of the research program of the Stichting voor Fundamenteel Onderzoek der Materie (Foundation for Fundamental Research on Matter) and was made possible by financial support from the Nederlandse Organisatie voor Zuiver Wetenschappelijk Onderzoek (Netherlands Organization for the Advancement of Pure Research).

\section{References}

[1] J.F. Ziegler, Nucl. Instr. and Meth. B6 (1985) 270.

[2] P.F. Byrne, N.W. Cheung, S. Tam, C. Hu, Y.C. Shih, J. Washburn and $M$. Strathman, in: Ion Implantation and Ion Beam Processing of Materials, eds. G.K. Hubler et al. (North-Holland, Amsterdam, 1984) p. 253.

[3] S. Mader, in: Ion Implantation Techniques, ed. H. Ryssel and H. Glawischning (Springer Verlag, Berlin, 1982) p. 299.

[4] L.C. Feldman, J.W. Maycr and S.T. Picraux, Materials Analysis by Ion Channeling (Academic Press, New York, 1982). 
[5] L. van der Zwan and K.W. Geiger, Nucl. Phys. A246 (1975) 93.

[6] J.K. Bair and F.X. Haas, Phys. Rev. C7 (1973) 1356.

[7] P. Sperr, H. Spieler, M.R. Maier and D. Evers, Nucl. Instr. and Meth. 116 (1974) 55.

[8] F. Ajzenberg-Selove, Nucl. Phys. A268 (1976) 1.

[9] J.F. Ziegler, Helium Stopping Powers and Ranges in All Elements (Pergamon Press, New York, 1977).

[10] P.J.M. Smulders, Nucl. Instr. and Meth. B14 (1986) 234.

[11] H. Kudo, Phys. Rev. B18 (1978) 5995.

[12] D.K. Brice, Ion Implantation Range and Energy Deposition Distributions, vol. 1 (Plenum, New York, 1975).

[13] F.H. Eisen, G.J. Clark, J. Bøttiger and J.M. Poate, Radiat. Eff. 13 (1972) 93.

[14] J. Bottiger and F.H. Eisen, Thin Solid Films 19 (1973) 239.

[15] D.C. Santry and R.D. Werner, Nucl. Instr. and Meth. 178 (1980) 523 .

[16] D.C. Santry and R.D. Werner, Nucl. Instr. and Meth. B1 (1984) 13.

[17] S. Oosterhoff, Thesis (unpublished), Twente University of Technology, Enschede, The Netherlands (1986).
[18] W.K. Hofker, H.W. Werner, D.P. Oosthoek and N.J. Koeman, Appl. Phys. 4 (1974) 125.

[19] J. Narayan and J. Fletcher, in: Defects in Semiconductors, eds., I. Narayan and T.Y. Tan (North-Holland, Amsterdam, 1981) p. 191.

[20] W. Mayer, D. Grasse and J. Peisl, Radiat Eff. 84 (1985) 107.

[21] B.C. Larson and J.F. Barhorst in: Defects in Semiconductors, eds., J. Narayan and T.Y. Tan (North-Holland, Amsterdam, 1981) p. 151.

[22] J.E. Westmoreland, J.W. Mayer, F.H. Eisen and B. Welch, Appl. Phys. Lett. 15 (1969) 308.

[23] D. Fink, J.P. Biersack, H.D. Carstanjen, F. Jahnel, K. Muller, H. Ryssel and A. Osei, Radiat. Eff. 77 (1983) 11.

[24] A. Fukuhara and Y. Takano, Acta Crystallogr. A33 (1977) 137.

[25] R.W. Bicknell and R.M. Allen, Radiat. Eff. 6 (1970) 45.

[26] J. Washburn, in: Defects in Semiconductors, eds, J. Narayan and T.Y. Tan (North-Holland, Amsterdam, 1981) p. 209. 